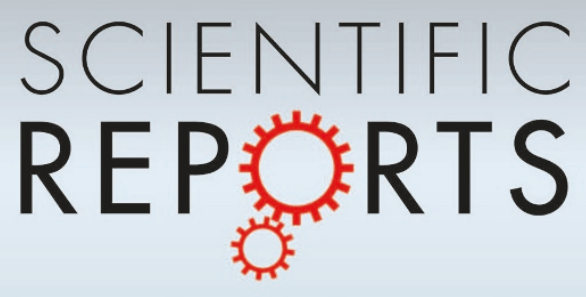

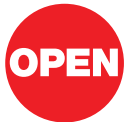

SUBJECT AREAS: MOLECULAR MODELLING

PATHOLOGY

NEUROSCIENCE

NEURODEGENERATION

Received

31 July 2012

Accepted

1 November 2012

Published

30 November 2012

Correspondence and requests for materials should be addressed to B.D. (benjamin. dehay@u-bordeaux2.

\section{Age-dependent $\alpha$-synuclein aggregation in the Microcebus murinus lemur primate}

\author{
Marie-Hélène Canron ${ }^{1,2}$, Martine Perret ${ }^{3}$, Anne Vital ${ }^{1,2}$, Erwan Bézard ${ }^{1,2} \&$ Benjamin Dehay ${ }^{1,2}$
}

'Univ. de Bordeaux, Institut des Maladies Neurodégénératives, UMR 5293, F-33000 Bordeaux, France, ${ }^{2}$ CNRS, Institut des Maladies Neurodégénératives, UMR 5293, F-33000 Bordeaux, France, ${ }^{3}$ CNRS UMR 7179, MNHN, Département Ecologie et gestion de la biodiversité, Brunoy, France.

Since age-dependent deposition of Aß-amyloid has been reported in the Microcebus murinus, we posited that this animal could as well be a model of age-related synucleinopathy. We characterized the distribution of A $\beta$-amyloid, $\alpha$-synuclein and two of its modified forms in the brain of Microcebus murinus aged from 1.5 to 10 years. Intracytoplasmic $\alpha$-synuclein aggregates were observed only in aged animals in different brain regions, which were also phospho-Ser129 and nitrated $\alpha$-synuclein immunoreactive. Age-dependent $\alpha$-synuclein aggregation occurs spontaneously in mouse lemur primates. Microcebus murinus may provide a model to study age-associated $\alpha$-synucleinopathy and for testing putative therapeutic interventions for both Alzheimer's and Parkinson's diseases.
A nimal models are an essential tool for basic pathophysiological research, age-related research as well as drug development and compound testing in neurodegenerative diseases ${ }^{1-3}$. So far, most of animal studies on neurodegenerative disorders are based on transgenic mice or rats. Although very useful to unravel cellular mechanisms associated to neuronal cell death during the time course of the disease, no mammalian model recapitulates the full spectrum required age-dependent neurodegeneration, especially in Parkinson disease $(\mathrm{PD})^{4,5}$. The aggregation-prone protein, $\alpha$-synuclein, has been implicated in various neurodegenerative disorders by participating in abnormal protein depositions, such as PD, dementia with Lewy bodies (DLB) and multiple system atrophy (MSA). The absence of adequate in vivo experimental models of synucleinopathy has severe repercussions for therapeutic intervention success ${ }^{5}$. Natural or ecological models would be an alternative to model pathological accumulation of $\alpha$-synuclein. Of interest, among primate models, Microcebus murinus might be a very good candidate to study age-associated synucleinopathy ${ }^{6,7}$. The Microcebus murinus, a small lemurian primate, also referred to as the mouse lemur, has been described as a useful primate model for human cerebral aging and Alzheimer disease ${ }^{8}$. In mouse lemurs, histological studies have shown cerebral morphological and neuropathological alterations associated to ageing, including abundant $\mathrm{A} \beta$ amyloid plaques, Tau pathology, and atrophy of brain areas such as the cortex, the hippocampus, the thalamus and hypothalamus, the basal ganglia and the cerebellum ${ }^{9-12}$. In addition, as in humans, magnetic resonance imaging has corroborated age-associated cerebral atrophy and iron accumulation ${ }^{13,14}$. Based on these studies, we assessed the occurrence of spontaneous $\alpha$-synuclein accumulation in the mouse lemur brain at different ages. Thus, the present study focuses on Microcebus murinus as a potential model for the study of age-associated synucleinopathy.

\section{Results}

We characterized the regional distribution of immunopositivity for misfolded proteins implicated in neurodegenerative diseases, such as $A \beta$ deposits and $\alpha$-synuclein aggregates (Fig. 1A-B). Intracytoplasmic $\alpha$-synuclein aggregates were observed only in old animals in the anterior olfactory nucleus, cortex, and regions implicated in $\mathrm{PD}$, such as the substantia nigra pars compacta ( $\mathrm{SNpc}$ ) and the striatum (Fig. 1D-F-H) and not in young individuals (Fig. 1C-E-G). An antibody that specifically recognizes phospho-Ser129 was used to determine whether neuronal cell bodies in the mouse lemur contain this modified form of $\alpha$-synuclein and whether $\alpha$ synuclein phosphorylation is enhanced by aging. Phospho-Ser129 $\alpha$-synuclein immunoreactive neurons were found in the cerebellum, hippocampus, thalamus, red nucleus, olfactory tubercle, cortex, $\mathrm{SNpc}$ and striatum of old mouse lemur primates (Fig. 1J-L-N), whereas no immunostaining was detected in young animals (Fig. 1I-KM). Double immunofluorescence examination revealed that phosphor-Ser129 $\alpha$-synuclein increases occurred within SNpc dopaminergic neurons in aged animals (Fig. 2A). Other post-translational modification may occur in $\alpha$-synuclein concerns nitration. Midbrain sections from young and old animals were therefore stained with an 

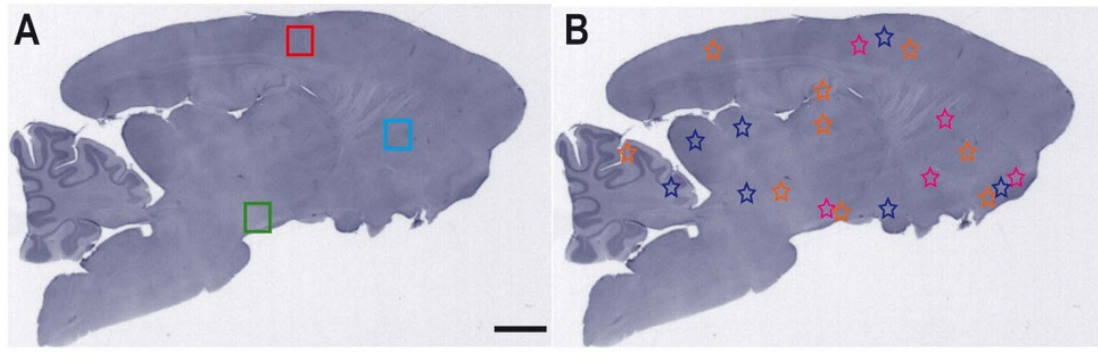

$\alpha$-synuclein LB509

P-Ser129 $\alpha$-synuclein

BA4 amyloid
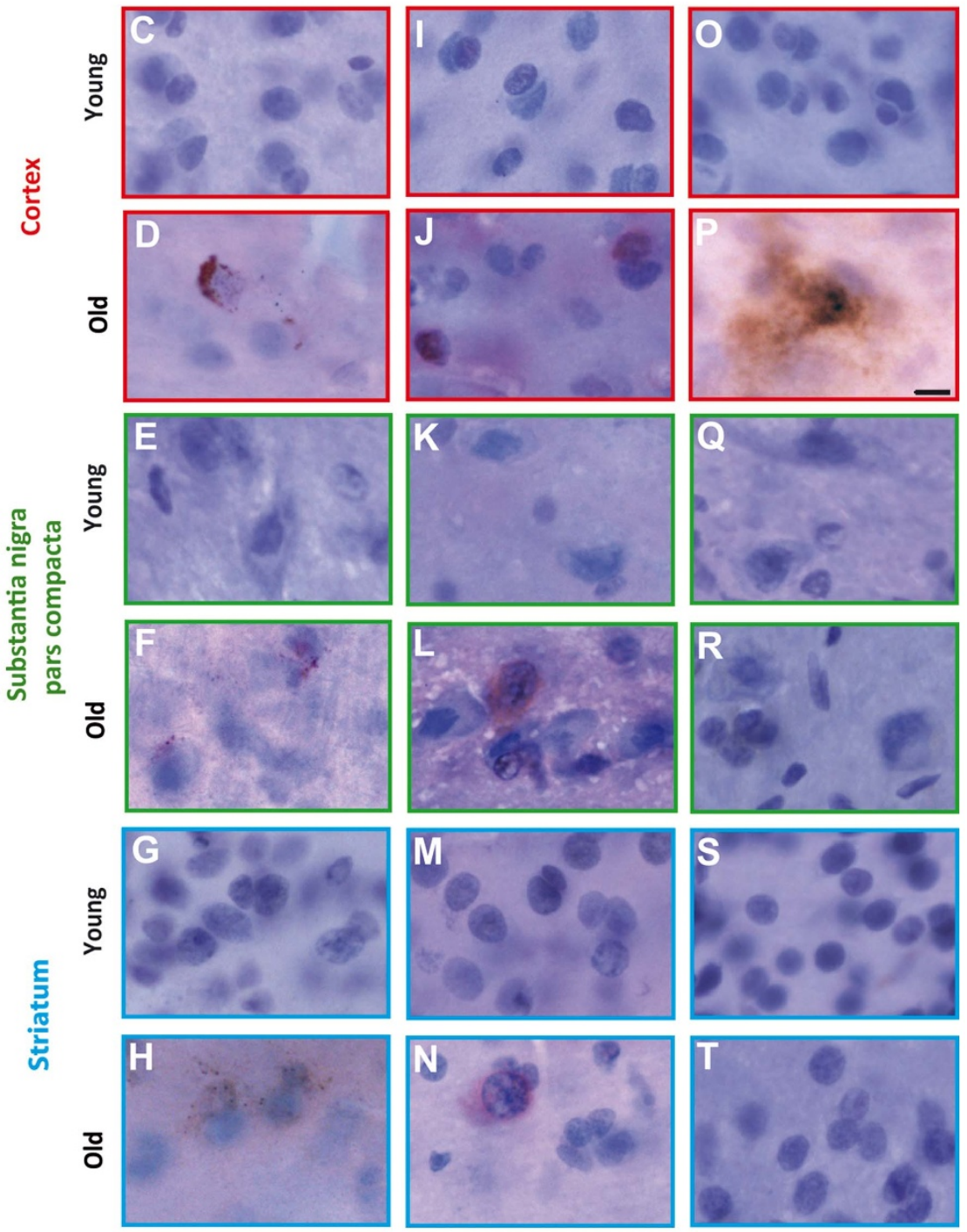

Figure $1 \mid$ Distribution of synuclein and amyloid pathology in mouse lemur brains. (A) Sagittal section of mouse lemur stained with Mayer's hemalum showing midbrain and forebrain regions. Scale bar $=2 \mathrm{~mm}$. (B) Sagittal section of mouse lemur stained with Mayer's hemalun showing distribution of $\beta$ A4 (purple stars), $\alpha$-synuclein (pink stars), Phospho-Ser129 $\alpha$-synuclein (orange stars) immunoreactive regions. (C-H) Magnifications of selected $\alpha$ synuclein positive regions in young and old animals boxed in A: cortex (C-D), SNpc (E-F), striatum (G-H). (I-N) Magnifications of selected PhosphoSer129 $\alpha$-synuclein positive regions in young and old animals boxed in A: cortex (I-J), SNpc (K-L), striatum (M-N). (O-T) Magnifications of selected $\beta A 4$ positive regions in young and old animals boxed in A: cortex (O-P), SNpc (Q-R), striatum (S-T); Scale bar $=10 \mu \mathrm{m}$.

anti-nitrated $\alpha$-synuclein antibody to detect potential age-related modifications. Similar to the results with phosphorylated $\alpha$-synuclein, immunoreactivity for nitrated $\alpha$-synuclein was slightly increased within nigral neurons of aged animals (Fig. 2B).

Brain sections were immunostained with antibody against $A \beta$ amyloid as a control for proteinopathy. $A \beta$ deposits were observed in many brain regions, such as cortex, cerebellum, inferior and superior colliculi, and anterior olfactory nucleus (Fig. 1B). Diffuse plaques were strongly positive for $A \beta$-amyloid in the cortex of old animals (Fig. 1P) whereas no $A \beta$ deposits were found in young animals (Fig. 1O). We did not observe $\beta A 4$-amyloid immunostaining either in the SNpc or in the striatum whatever the age of the animals.

\section{Discussion}

The purpose of this study was to investigate age-related accumulation of $\alpha$-synuclein. We found that it specifically occurs as mouse 
A
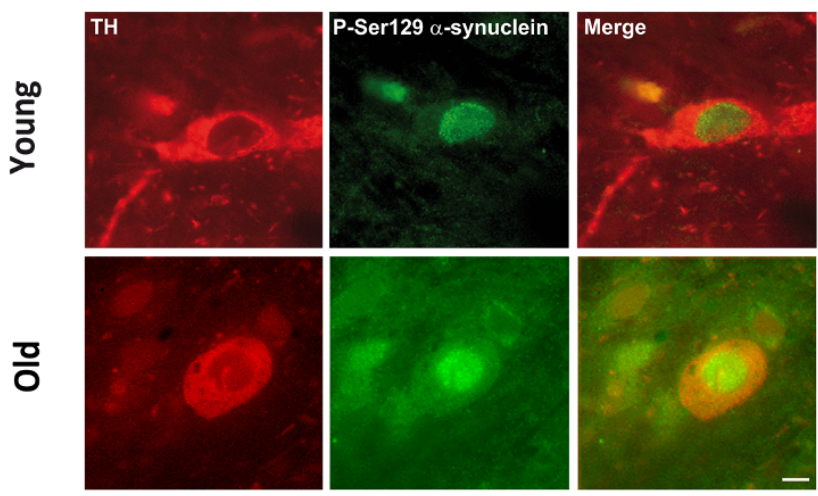

B
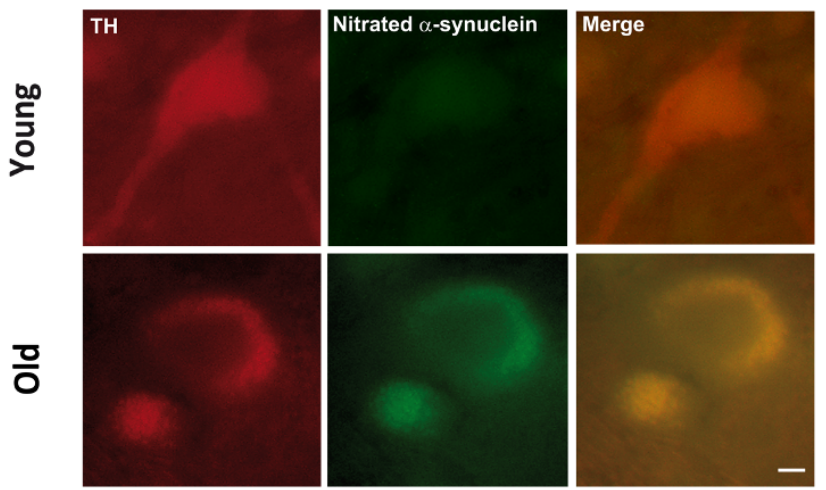

Figure $2 \mid$ Phosphorylated and nitrated $\alpha$-synuclein immunoreactivity is enhanced in the substantia nigra of old $(n=4)$ as compared with young $(n=3)$ mouse lemur. (A) Representative midbrain sections from young and old mouse lemur co-immunostained with tyrosine hydroxylase $(\mathrm{TH}$, red) and an anti-phosphorylated $\alpha$-synuclein (P-Ser129 $\alpha$-synuclein, green). The merged image shows colabeling within a nigral neuron. (B) Double immunofluorescence for nitrated $\alpha$-synuclein (green) in THpositive neurons (red) in young and old animals. The merged image shows coimmunoreactivity within a nigral neuron. Scale bar for panels A and $\mathrm{B}=10 \mu \mathrm{m}$.

lemur primates aged, and within regions implicated in $\mathrm{PD}$, such as the SNpc.

Diffuse extracellular cortical A $\beta$-amyloid deposits were detected in every aged Microcebus brains, as previously reported by Bons and colleagues $^{9,10}$. Indeed, these histological changes consisted in a large number of senile plaques composed of degenerated neurites sometimes surrounding an amyloid plaque. In addition to the "classic" $\mathrm{A} \beta$-amyloid pathology, aging was also accompanied by the occurrence of $\alpha$-synuclein in neurons of many brain areas and of interest in nigral cell bodies. Post-translational modifications of $\alpha$-synuclein affect the biochemical and structural properties of the protein, and might promote aggregation and toxicity ${ }^{15}$. Phosphorylation at Ser129 has been reported to be the predominant modification of $\alpha$-synuclein in Lewy bodies ${ }^{16}$. Of interest, immuno-expression of phosphoSer129 and nitrated $\alpha$-synuclein confirmed the formation of phosphorylated and nitrated protein within dopaminergic neurons. Such striking relationship between aging and misfolded protein deposition in the brain of mouse lemur primates strongly suggests that this species carries a specific and unique potential for the further study of ecologically-occurring proteinopathies.

In conclusion, these data show for the first time that i) $\alpha$-synuclein aggregation can occur spontaneously with aging in mouse lemur primates and that ii) Microcebus murinus may provide an ecological model for the study of age-associated synucleinopathy. This spontaneous animal model may provide an ideal system for understanding the mechanisms and the dynamic evolution of ageing and related proteinopathies as well as a model into which identifying predictive biomarkers and testing therapeutic interventions for $\mathrm{AD}$ and PD.

\section{Methods}

Animals. All mouse lemurs were housed in social cages with their mothers under controlled conditions of humidity, temperature, and light (12-h light/12-h dark cycle, lights on at $8.00 \mathrm{am}$ ); food and water were available ad libitum. Experiments were carried out in accordance with European Communities Council Directive of 3 June $2010(2010 / 6106 / \mathrm{EU})$ for care of laboratory animals in the breeding colony established at Brunoy (France, agreement A91.114.1) from a stock originally caught near the south coast of Madagascar, in 1965-1972. These studies were approved by the Ministry of Education and Science and the University of Bordeaux ethical committee. We studied 3 brains removed from young Microcebus murinus ( 2 males and 1 female) between 1.42 to 3.07 years old and 4 brains from old individuals ( 2 males and 2 females) aged 8.21 and 10.32 years old. All the animals were provided from the laboratory breeding colony at Brunoy (France, Perret M.).

Brain pathology. Neuropathological studies of mouse lemur brains were performed with procedures and antibodies used in human pathology, as described below.

Brains were fixed by immersion in $4 \%$ paraformaldehyde in $0.1 \mathrm{M}$ Phosphate buffer ( $\mathrm{pH}$ 7.4). One fixed hemi-brain was sectioned on a vibratome at $50 \mu \mathrm{m}$ on the sagittal plane. Immunohistochemistry was performed with a horseradish peroxydase (HRP) method with the following antibodies: anti $\alpha$-synuclein clone LB509, a mouse monoclonal antibody (1:100 dilution; Invitrogen, Cergy-Pontoise, France); anti PSer129, a mouse monoclonal antibody against phospho-Ser129 $\alpha$-synuclein (1:500; Wako); and anti $\mathrm{A} \beta$-amyloid clone $6 \mathrm{~F} / 3 \mathrm{D}$, a mouse monoclonal antibody $(1: 100$; Dako, Trappes, France). Briefly, the sections were rinsed in PBS, permeabilized with PBS containing $0.3 \%$ triton $\mathrm{X}-100$. For immunostaining with anti $\mathrm{A} \beta$-amyloid and anti PSer129, sections were pretreated with formic acid (80\%) and next with proteinase K (Dako, Trappes, France) for A $\beta$-amyloid immunolabelled sections only to enhance immunoreactivities. To block non specific reactions, sections were then exposed to a universal blocking reagent (Biogenex) for LB509 $\alpha$-synuclein and PSer129 or to PBS containing 10\% bovine albumin serum for 30 min for anti A $\beta$ amyloid. Incubation with primary antibodies lasted overnight at $4{ }^{\circ} \mathrm{C}$. Subsequently, the sections were transferred in $3 \% \mathrm{H}_{2} \mathrm{O}_{2}$ in PBS for $15 \mathrm{~min}$ to inhibit endogenous peroxidases and treated with a ready-to-use goat anti mouse En Vision-HRP enzyme conjugate (Dako, Trappes, France) for $40 \mathrm{~min}$. The highly sensitive diaminobenzidin plus $(\mathrm{DAB}+)$ and the 3 -amino-9 ethyl carbazol plus (AEC+) (both from Dako, Trappes, France) were used as substrates chromogens. Finally, sections were counterstained with Mayer's hemalum and mounted in an aqueous medium for microscopy. Immunohistochemical negative controls were performed by omission of the primary antibody. Double immunofluorescence was performed with a mouse monoclonal phospho-Ser $129 \alpha$-synuclein (1:500; Wako) and a rabbit polyclonal anti tyrosine hydroxylase (TH) $(1: 4000$; Millipore) as well as a mouse monoclonal nitrated $\alpha$-synuclein $(1: 100$, clone Syn 505 ; Invitrogen) and TH. For immunostaining with anti-nitrated $\alpha$-synuclein, sections were pretreated 5 minutes with formic acid (80\%). Secondary antibodies were Alexa Fluor 488-labeled goat anti-mouse (Invitrogen) for phospho-Ser129 $\alpha$-synuclein or goat anti-mouse En Vision-HRP enzyme conjugate followed by a dylight- 488 conjugated goat anti-horseradish peroxydase (Jackson ImmunoResearch Laboratories) for anti-nitrated $\alpha$-synuclein. To localize TH in dopaminergic neurons, Alexa Fluor 568-labeled donkey anti-rabbit (Invitrogen) was used.

1. Moore, D. J. \& Dawson, T. M. Value of genetic models in understanding the cause and mechanisms of Parkinson's disease. Curr Neurol Neurosci Rep 8, 288-296 (2008).

2. Jenner, P. Functional models of Parkinson's disease: a valuable tool in the development of novel therapies. Ann Neurol 64 Suppl 2, S16-29 (2008).

3. Meredith, G. E., Sonsalla, P. K. \& Chesselet, M. F. Animal models of Parkinson's disease progression. Acta Neuropathol 115, 385-398 (2008).

4. Dehay, B. \& Bezard, E. New animal models of Parkinson's disease. Mov Disord 26, 1198-1205 (2011).

5. Bezard, E., Yue, Z., Kirik, D. \& Spillantini, M. G. Animal models of Parkinson's disease: Limits and relevance to neuroprotection studies. Mov Disord (2012).

6. Austad, S. N. \& Fischer, K. E. The development of small primate models for aging research. ILAR J 52, 78-88 (2011).

7. Languille, S. et al. The grey mouse lemur: a non-human primate model for ageing studies. Ageing Res Rev 11, 150-162 (2012).

8. Bons, N., Rieger, F., Prudhomme, D., Fisher, A. \& Krause, K. H. Microcebus murinus: a useful primate model for human cerebral aging and Alzheimer's disease? Genes Brain Behav 5, 120-130 (2006).

9. Bons, N., Mestre, N. \& Petter, A. Senile plaques and neurofibrillary changes in the brain of an aged lemurian primate, Microcebus murinus. Neurobiol Aging 13, 99-105 (1992).

10. Bons, N. et al. Identification of amyloid beta protein in the brain of the small, short-lived lemurian primate Microcebus murinus. Neurobiol Aging 15, 215-220 (1994).

11. Kraska, A. et al. Age-associated cerebral atrophy in mouse lemur primates. Neurobiol Aging 32, 894-906 (2011). 
12. Picq, J. L. Aging affects executive functions and memory in mouse lemur primates. Exp Gerontol 42, 223-232 (2007).

13. Dhenain, M. et al. MRI description of cerebral atrophy in mouse lemur primates. Neurobiol Aging 21, 81-88 (2000).

14. Dhenain, M. et al. Cerebral T2-weighted signal decrease during aging in the mouse lemur primate reflects iron accumulation. Neurobiol Aging 19, 65-69 (1998).

15. Oueslati, A., Fournier, M. \& Lashuel, H. A. Role of post-translational modifications in modulating the structure, function and toxicity of alphasynuclein: implications for Parkinson's disease pathogenesis and therapies. Prog Brain Res 183, 115-145 (2010)

16. Anderson, J. P. et al. Phosphorylation of Ser-129 is the dominant pathological modification of alpha-synuclein in familial and sporadic Lewy body disease. J Biol Chem 281, 29739-29752 (2006).

\section{Acknowledgements}

This work was supported by ANR-08-MNP-018 MCHPRIMAPARK Agence Nationale de la Recherche grants (EB), Biothèque Primate - Centre National de la Recherche Scientifique Life Sciences Department (EB), a post-doc fellowship from Fondation pour la Recherche
Médicale (BD) and a FP7-PEOPLE-2009-RG (BD). The Université Bordeaux Segalen and the Centre National de la Recherche Scientifique provided the infrastructural support.

\section{Author contributions}

B.D. and E.B. designed research; M.H.C., M.P., A.V. performed research; M.H.C., M.P., A.V., B.D. and E.B. analyzed data; B.D. and E.B. wrote the main manuscript text and B.D and M.H.C. prepared figures. All authors reviewed the manuscript.

\section{Additional information}

Competing financial interests: EB is Chief Scientific Officer of Motac neuroscience Ltd. All other authors reported no biomedical financial interests or potential conflicts of interest.

License: This work is licensed under a Creative Commons

Attribution-NonCommercial-NoDerivs 3.0 Unported License. To view a copy of this

license, visit http://creativecommons.org/licenses/by-nc-nd/3.0/

How to cite this article: Canron, M.H., Perret, M., Vital, A., Bézard, E. \& Dehay, B.

Age-dependent $\alpha$-synuclein aggregation in the Microcebus murinus lemur primate. Sci. Rep 2, 910; DOI:10.1038/srep00910 (2012). 